\title{
Antenna for humidity sensor using split ring resonator
}

\author{
Siti Aminah Md Akhir ${ }^{1}$, Siti Zuraidah Ibrahim² ${ }^{2}$ Nurshamimi Rosli ${ }^{3}$, Aini Syuhada Md Zain ${ }^{4}$, \\ Nurehansafwanah Khalid ${ }^{5}$ \\ 1,2,3,5 Advanced Communication Engineering (ACE) Centre, Universiti Malaysia Perlis, Malaysia \\ ${ }^{4}$ School of Computer and Communication Engineering, Universiti Malaysia Perlis, Malaysia
}

\begin{tabular}{|c|c|}
\hline Article Info & ABSTRACT \\
\hline Article history: & This paper presents an antenna as a humidity sensor application to control and \\
\hline Received Sep 12, 2018 & $\begin{array}{l}\text { monitor the quality of humidity. A passive sensor tag with low cost solution is } \\
\text { suggested where it implemented on a low-cost substrate, FR4. A capacitive }\end{array}$ \\
\hline Revised Nov 2, 2018 & sensor that works from 0 to $1.5 \mathrm{GHz}$ is implemented in the design. The \\
\hline Accepted Nov 25, 2018 & $\begin{array}{l}\text { capacitive sensor is designed based on a small antenna loaded with a Split ring } \\
\text { resonator (SRR). To sense the humidity, the SRR resonator is coated with }\end{array}$ \\
\hline Keywords: & $\begin{array}{l}\text { Kapton, placed on the top of the resonator. To validate the proposed method, } \\
\text { a prototype was fabricated. The comparison between simulated and measured }\end{array}$ \\
\hline Humidity Sensor & efficient is presented. \\
\hline
\end{tabular}

Metamaterial

Small loop antenna

Split ring resonator (SRR)

Copyright (C) 2019 Institute of Advanced Engineering and Science. All rights reserved.

\section{Corresponding Author:}

Siti Zuraidah Ibrahim,

Advanced Communication Engineering (ACE) Centre,

School of Computer and Communication Engineering,

Universiti Malaysia Perlis,

Pauh Main Campus, 02600 Arau, Perlis, Malaysia.

Email: sitizuraidah@unimap.edu.my

\section{INTRODUCTION}

A humidity sensor will measure the relative humidity $(\mathrm{RH})$ of water vapour that presence in the air. RH is most commonly used unit for humidity measurement and express in term of percentage (\%). Humidity sensors are widely used in home, workplaces, autos and etc as monitoring and preventive measure since humid environment will give an effect to consumers. In industries, measurement of humidity is critical since it might influence the cost of the item, wellbeing and safety of work force. For human comfort and industrial processes, humidity sensing plays an important role in control systems. Controlling humidity is of paramount importance in numerous modern and local applications. For local applications, humidity control is required for living environment in buildings and cooking control for microwave ovens. In every single application and many others, humidity sensors are employed to provide an indication of the moisture level in the environment. Humidity sensor is divided into two categories which employ capacitive and resistive sensing. Other than that, passive sensor allows a certain quantity to be measured without any power supply to operate. Passive and wireless sensor rapidly increases since they represent a low-cost implementation that not required any battery or power supply.

Electromagnetic Metamaterials (MTMs) are artificially engineered structures having properties not readily available in nature. These MTM are called Left Hand Material (LHM) [1]. Typically, a single metallic metamaterial inclusion can be considered as an LC resonant circuit with its inductance and capacitance influenced by its shape and dimensions. These resonators can collectively exhibit macroscopically observed effective values of permittivity and permeability that are not found in natural materials. A Split-ring resonator (SRR) is one of the MTM. On the other hand, metamaterial inspired antennas have been proved to serve as chipless sensing tags [2] in which they are useful for causing resonance and increasing sensitivity [3]. 
Antenna sensor works by sensing humidity using capacitance principle and measure the reflection coefficient, $\Gamma$. When the amount of water vapour changes in humidity level, the antenna will fill with content of water vapour in surrounding environment. Later on, antenna sensor will produce a resonant frequency shift when the sensors measure any changes in capacitance. Level of water vapor will influence the dielectric permittivity and change of reflection coefficient, $\Gamma$ usually depends on amount of water vapor in air. However only few SRR-based sensors have been reported: In [4], the idea of a capacitive sensor has been presented based on a rectangular SRR with metallic ring that acts as an inductor. It operates between frequency ranges of 8 to $12 \mathrm{GHz}$ on Rogers RO4003 substrate. The dielectric substrate and gap behave as a capacitance. This paper explored the use of MTM to sense thin film and their application in sensing properties. Three approaches are proposed which is using thin film sensing using SRR in gap, under ring and as an over layer. The result shows the coupling capacitance between the ring and the gap capacitance both change after adding a dielectric layer on the ring. These changes are proportional to the permittivity of the dielectric layer. The amount of the shift depends on the permittivity of the dielectric layer. In [5], the authors proposed a moisture sensor operating between 0.5 to $2 \mathrm{GHz}$ based on SRR unit cell. The prototype was fabricated on Taconic CER-10 substrate. The performance of proposed sensor is determined for soil moisture varying from 2 to $20 \%$. The resonant frequency shows that the higher soil moisture causes an increase of soil dielectric constants which lead to higher effective permittivity and cause a decrease of resonant frequency of the sensor. Apart from that, the drawback of some of proposed sensor is relatively small insertion loss at the resonant frequency especially in case of high soil moisture content.

In this paper we present a passive sensor with capacitive sensing capability based on miniaturized antenna with MTM loading approach. The sensor is build by a small loop antenna loaded with Split ring resonator (SRR) which reduces the tag dimension. The sensor conceived a single layer design and it fully compatible with printing fabrication process. Simulation and measurement result of sensing tag prototype on FR4 substrate is presented. The sensing capability is demonstrated by coating the SRR ring with Kapton in order to make it work as humidity sensor. The result show good sensitivity for sensor implemented. Kapton Polyimide exhibits excellent sensitivity to environment RH as replicated in the experimental results presented in this paper. These features make this antenna sensor a potential device for low cost, compact and superior sensitive passive humidity sensing. Thus it can revolutionize the cold chain management, storage and transport of perishable goods, pharmaceuticals and chemicals.

\section{DESIGN TECHNIQUE}

The antenna concept is related to the small antenna approach with MTM loading and it is called near field parasitic elements [6]. Thus, SRR is coupled to an electrically small loop antenna. The coupling among these two structures is the mechanism that increases the radiation efficiency by enhancing the loop magnetic near field. The proposed SRR antenna is designed on one sided FR4 substrate materials where it copper on the top layer denoted as patch antenna. The relative dielectric constant, $\varepsilon_{r}$ of the board is 4.3 with thickness, $h$ is $1.6 \mathrm{~mm}$ and dissipation factor, $\tan \delta$ is 0.02 . The size of substrate is $35 \mathrm{~mm} \times 35 \mathrm{~mm}$. Figure 1 shows the physical of small loop antenna loaded with SRR metamaterial.

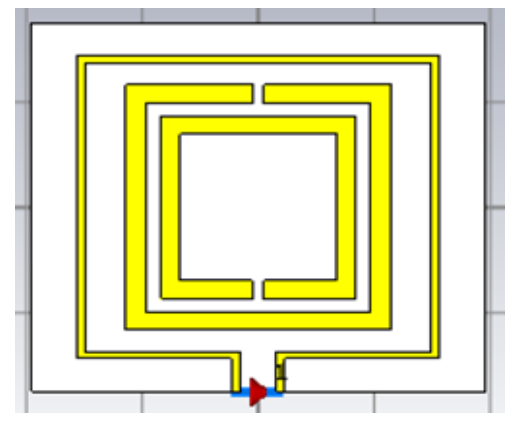

Figure 1. Small loop antenna loaded with SRR without Kapton

The performance of proposed antenna is evaluated with and without using Kapton as a sensing material. In order to gain high sensitivity, Kapton is applied on top of SRR ring. A simulation was performed using $50 \Omega$ characteristic impedance by using Computer Simulation Technology (CST) software. This antenna 
is designed between $0 \mathrm{GHz}$ to $1.5 \mathrm{GHz}$ of frequency range. Noted that a new material with Kapton properties is required to be created in the CST library.

When a time varying magnetic field is applied perpendicular to the SRR surface, a current density is induced in a way that it is minimum at the gaps and maximum on the other side, accumulating opposite charges at the extremities of each ring and hence producing a high distributed capacitance. The gaps were made small to produce high quality factor that is convenient for sensing applications [7]. Table 1 and Figure 2 show the dimensions of the proposed layout.

Table 1. The Dimensions of Antenna

\begin{tabular}{ccc}
\hline Parameters & Descriptions & Size $(\mathrm{mm})$ \\
\hline$L s$ & Length of FR-4 substrate & 35 \\
$W s$ & Width of FR-4 substrate & 35 \\
$D 1$ & Length of outer ring & 31.2 \\
$D 2$ & Length of ring 2 & 23.9 \\
$D 3$ & Length of ring 3 & 17 \\
$G 1$ & Horizontal gap between ring 1 and 2 & 2.7 \\
$G 2$ & Horizontal gap between ring 2 and 3 & 1.8 \\
$G 3$ & Vertical gap between ring 1 and 2 & 2.5 \\
$S 1$ & Gap of open-ended of ring 1 & 3 \\
$S 2$ & Gap of open-ended ring 2/3 & 1 \\
$W$ & Width of copper outer ring & 0.7 \\
$W 1$ & Width of copper ring2 and & 1.75 \\
\hline
\end{tabular}

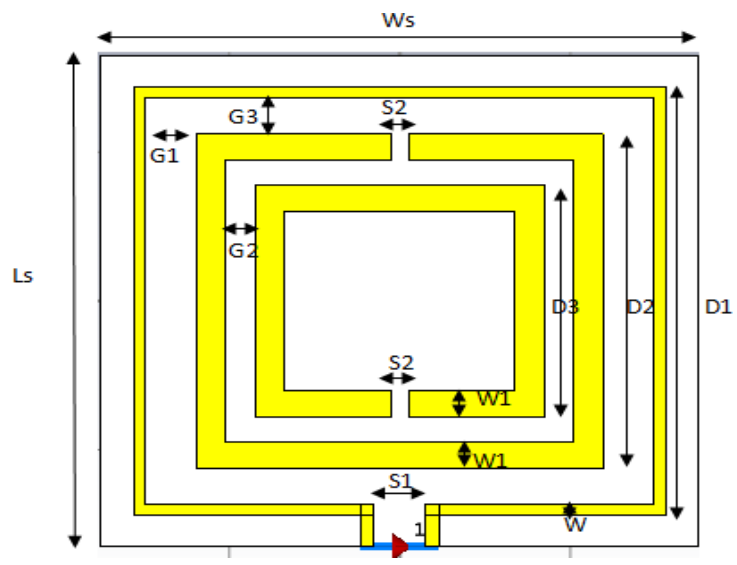

Figure 2. Layout of antenna dimension

Kapton is a sensing material type [8] will use as superstrate in this project. Superstrate is referring to another layer on top of the substrate [9]. Thus, need to create new material with kapton properties and add into library. By pick face the ring of patch and perform extrude function in CST and then the ring of patch will cover up by Kapton on top of the patch as a superstrate. Kapton has linear change with humidity permittivity and the changes of relative permittivity with humidity $(\mathrm{RH})$ given by

$$
\varepsilon_{r}=3.05+0.008 \times \mathrm{RH}
$$

Kapton is a polyimide film and this film is stable even in a wide range of temperature. In electronic manufacturing, kapton tape is usually used as protection layer on fragile component. Kapton operate as capacitive sensor for humidity because it has linear dielectric response. It has higher UL-94 flammability rating so it does not burn or melt. These properties allow kapton to be used at high and low temperature. Kapton $\mathrm{HN}$ used in application at temperature range from $-269^{\circ} \mathrm{C}$ to $400^{\circ} \mathrm{C}$ [8]. The relative dielectric constant, $\varepsilon_{r}$ of the Kapton is 3.4 with thickness, $h$ is $0.035 \mathrm{~mm}$. Figure 3 shows the layout of the antenna with Kapton on the top. 


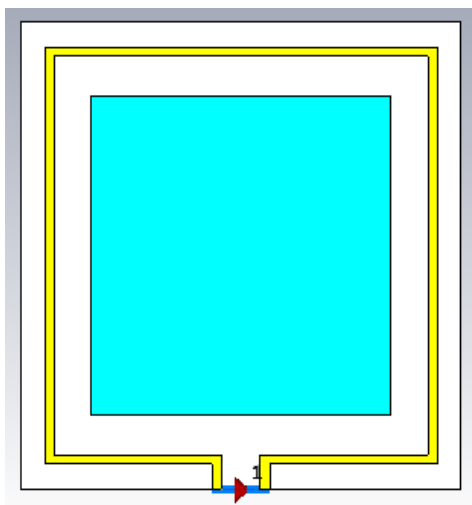

Figure 3. Small loop antenna loaded with SRR with Kapton

\section{RESULTS AND ANALYSIS}

To incorporate humidity sensing, the fabricated antenna is modified by putting Kapton film on top of the SRR resonator [10]. To validate the sensor operation, an experiment is performed using Vector Network Analyzer (VNA). Figure 4 shows a fabricated antenna coated with Kapton film on top of substrate.

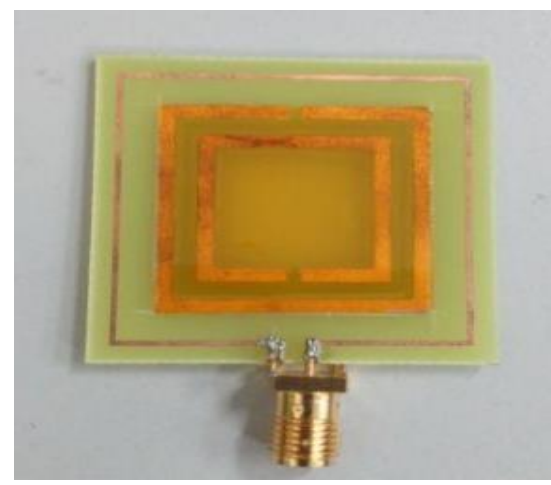

Figure 4. Fabricated antenna with Kapton

Simulation result of Split Ring Resonator (SRR) as humidity sensor is demonstrated in Figure 5 for both scheme, with and without using kapton.

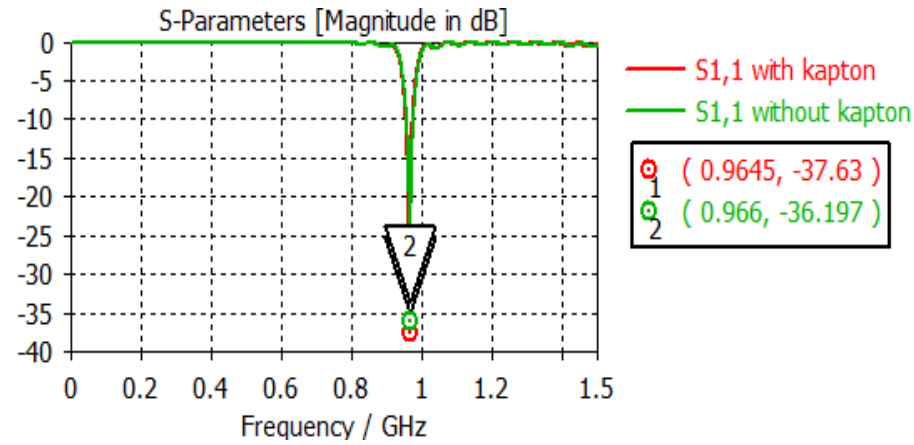

Figure 5. Simulation of reflection coefficient

From the S11 result, Figure 5 indicates the resonant frequency of antenna without Kapton is 0.966 $\mathrm{GHz}$ at -36.197 while the frequency of antenna with Kapton superstrate is $0.9645 \mathrm{GHz}$ at -37.63 . The result 
for antenna incorporated with Kapton presents comparable results in terms of reflection coefficient compared to the one without Kapton. This indicates that Kapton is suitable to integrate with the SRR without changing the original performances. Figure 6 demonstrates the operating bandwidth of antenna with and without Kapton at $-10 \mathrm{~dB}$.

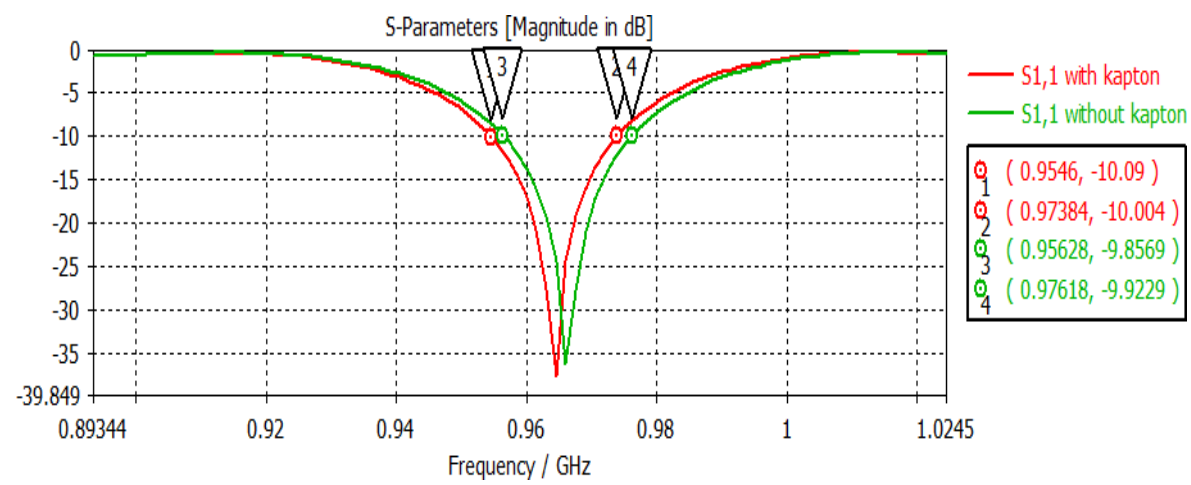

Figure 6. Simulated operational bandwidth at $10 \mathrm{~dB}$ return loss

By using (with) kapton the operating frequency ranging from lower frequency ( $\mathrm{fL}$ ) is $0.9546 \mathrm{GHz}$ to higher frequency $(\mathrm{fH})$ is $0.97384 \mathrm{GHz}$. Then, for simulation antenna without kapton shows that at $-10 \mathrm{~dB}$, the operating frequency ranging from lower frequency (fL) is $0.95628 \mathrm{GHz}$ to higher frequency (fH) is 0.97618 $\mathrm{GHz}$. The bandwidth for antenna with kapton is $1.995 \%$ which is from an extended of frequency $0.9546 \mathrm{GHz}$ until $0.97384 \mathrm{GHz}$ while the designed antenna without kapton which is the range of frequency covered from $0.95628 \mathrm{GHz}$ until $0.97618 \mathrm{GHz}$ has $2.06 \%$ of bandwidth. Bandwidth is used to describe the range of frequency which the antenna can receive energy, properly radiate and operate correctly. Figure 7 represents the comparison between simulation and measurement.

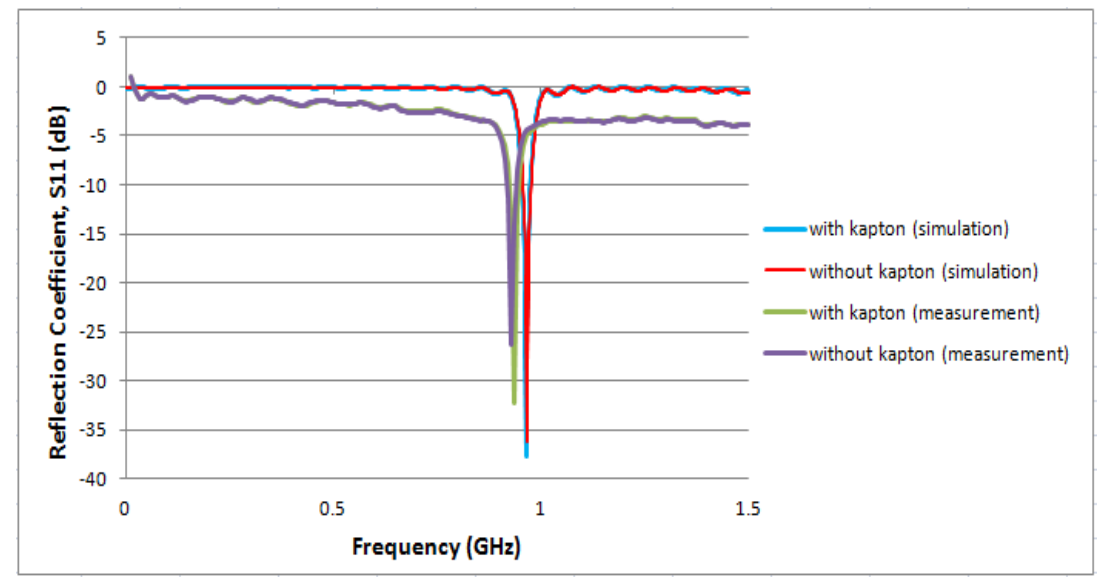

Figure 7. Comparison between simulated and measured reflection coefficient

As depicted in Figure 7, the minimum measured resonant frequency occurred at $0.955 \mathrm{GHz}$ for both with and without Kapton. From the measurement result the reflection coefficient is decreased compared to simulation result which is $-33.02 \mathrm{~dB}$ and $-37.69 \mathrm{~dB}$. However reflection coefficient performance for measured antenna is satisfied because it still below than $10 \mathrm{~dB}$. The decrement of reflection coefficient and frequency for measurement maybe due to fabrication process because it will affect the performance of implemented antenna.

Figure 8 indicate the measurement result for antenna as humidity sensor. Kapton film is applied on top of ring 2 and ring 3 of SRR as sensing application. 


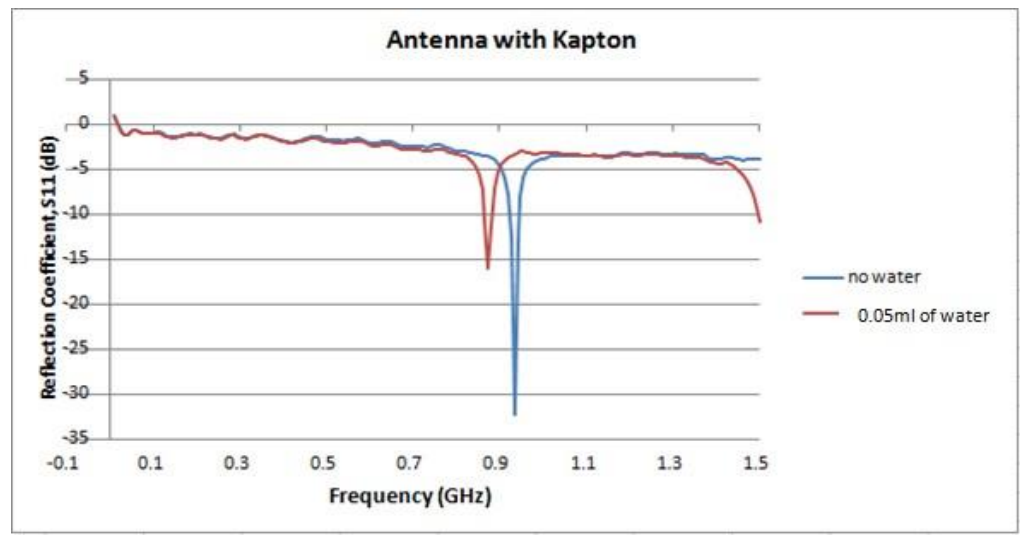

Figure 8. Reflection coefficient variation in response to relative humidity

The graph shows the measurement result of antenna sensor with no water and with water. The measurement was taken at room temperature which is $22.6^{\circ} \mathrm{C}$ with relative humidity $(\mathrm{RH})$ at $48.5 \%$. The variations of frequency occur when there is a change in temperature and humidity. This happened when $0.05 \mathrm{ml}$ of water is applied on top of Kapton. Thus this will affect the changes in capacitance value of SRR and after that frequency will start shifting to the lower frequency. When RH value increase, value of relative permittivity also increases and frequency will decrease. Note that Kapton has a linear change with humidity permittivity. The changes of relative permittivity with humidity at this RH are 148.313. This value can befound by using expression given in (1). Overall, these result shown that it can be accepted as antenna sensor for sensing application.

\section{CONCLUSION}

Humidity sensor is very useful for control and monitoring applications in industrial environments. However, in order to construct an intelligent sensor network, the sensor must be at low cost. In this paper we proposed a SRR antenna and the antenna concept related to the small antenna approach with metamaterial loading and coupled to small loop antenna. When SRR is coated with Kapton, the superstrate will react with surrounding humidity and when percentage of RH is increase, value of dielectric Kapton will increase too. Simulation result in CST shows a slight change of frequency and S11 result between SRR with kapton and without Kapton. The sensor proved to be suitable for passive sensing and also provide low cost solution fully compatible with printing fabrication process

\section{REFERENCES}

[1] S. S. Islam, et al., "The design and analysis of a novel split H-shaped Metamaterial for Multi-band microwave application," Materials, vol. 7(7), pp. 4994-5011, Feb 2011.

[2] C. Mandel, et al., "Metamaterial-inspired passive chipless radio frequency identification and wireless sensing," Annals of telecommunications, vol. 68 (7-8), pp. 385-399, 2013.

[3] M. Schubler, et al., "Metamaterial Inspired Microwave Sensors," IEEE Microwave Magazine, vol. 13(2), pp. 57-68, 2012.

[4] M. Labidi, et al., "Meta-metarials applications in thin film sensing and sensing liquids properties," Optics Express, vol. 19(S4), pp. A733-A739, 2011.

[5] G. Kitic, et al., "Soil moisture sensor based on metamaterial," Songklanakarin Journal Science of Technology, vol. 34(6), pp. 689-693, Dec 2012.

[6] Y. Dong and T. Itoh, "Metamaterial-Based Antennas," in Proceedings of the IEEE, vol. 100, no. 7, pp. 2271-2285, July 2012.

[7] H. Nornikman, et al., "Split ring Resonator structure on microstrip patch antenna and other microwave application design: A Review," Journal of Telecommunication, Electronic and Computer Engineering, vol. 7 (2), pp. 83-88, Dec 2015

[8] C. Li, et al., "Review of research status and development trends of wireless passive LC resonant sensor for harsh environment," Journal of Sensors (Basel), vol. 15(6), pp. 13097-13109, June 2015.

[9] R. A. Romero, et al., "A low cost passive wireless capacitive sensing tag based on split-ring resonator," 2015 IEEE International Instrumentation and Measurement Technology Conference (I2MTC) Proceedings, pp. 434-439, 2015

[10] E. Rufus, "Microstrip antenna on kapton substrate for strain sensing applications," 16th International Conference on Advanced Communication Technology, Pyeongchang, 2014, pp. 453-455. 


\section{BIOGRAPHIES OF AUTHORS}

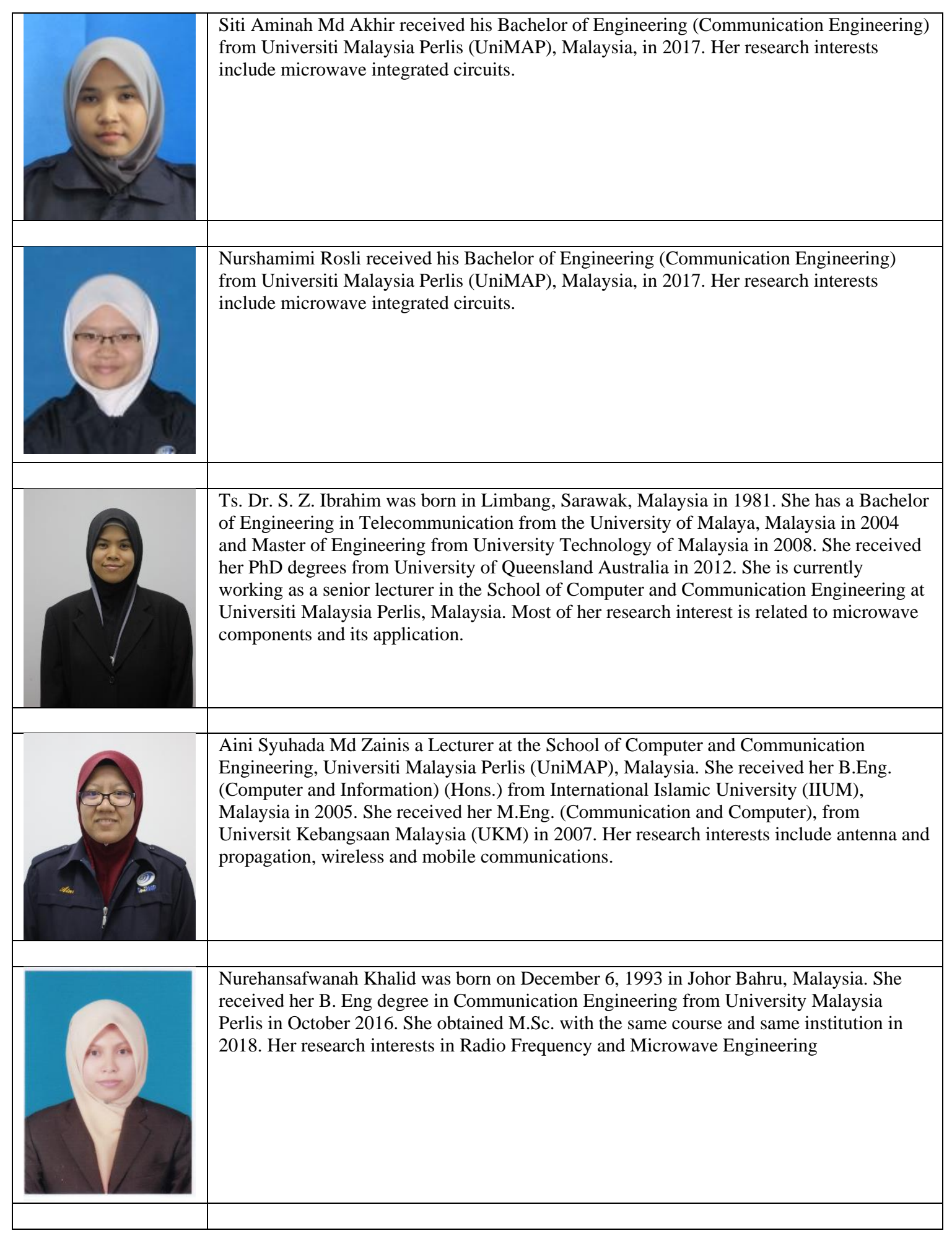

